\title{
Encrypted Data Hiding \& Retrieval of an Image using LSB Based on RBF Network
}

\author{
Amera Istiqlal Badran Abdulsattar M. Khidhir \\ amera_istiqlal@uomosul.edu.iq \\ College of Computer \\ Science and Mathematics \\ University of Mosul
}

Received on:2010/9/15

\author{
laheeb Mohammed Ibrahim \\ Laheeb_alzubaidy@yahoo.com \\ College of Computer \\ Science and Mathematics \\ University of Mosul
}

Accepted on:2010/11/10

\begin{abstract}
In this paper an image is hidden in another image using one of the hiding algorithms (Least Significant Bit) to produce the stego-cover image which used as an input with the cover to Radial basis function Network to produce the weights.

Cover is delivered once to the recipient who can use it for unlimited number of messages. The weights are delivered to the recipient for each hidden message as a key. The recipient uses the cover with the weights to unhide the message. So that this method include two levels of security. The first one is hiding the message in the cover to produce stego-cover image. The second one is ciphering the embedded image using RBF Neural Network. This Network is considered as a target and the input to the Neural Network is the cover image. Then the weights, which represent the encrypted information are reconstructed. The recipient can use RBF Network to unhide the message by having the stego-cover image then the message.
\end{abstract}

Matlab R2008a was used in this paper.

Keywords: Encryption, LSB algorithm, RBF Network.

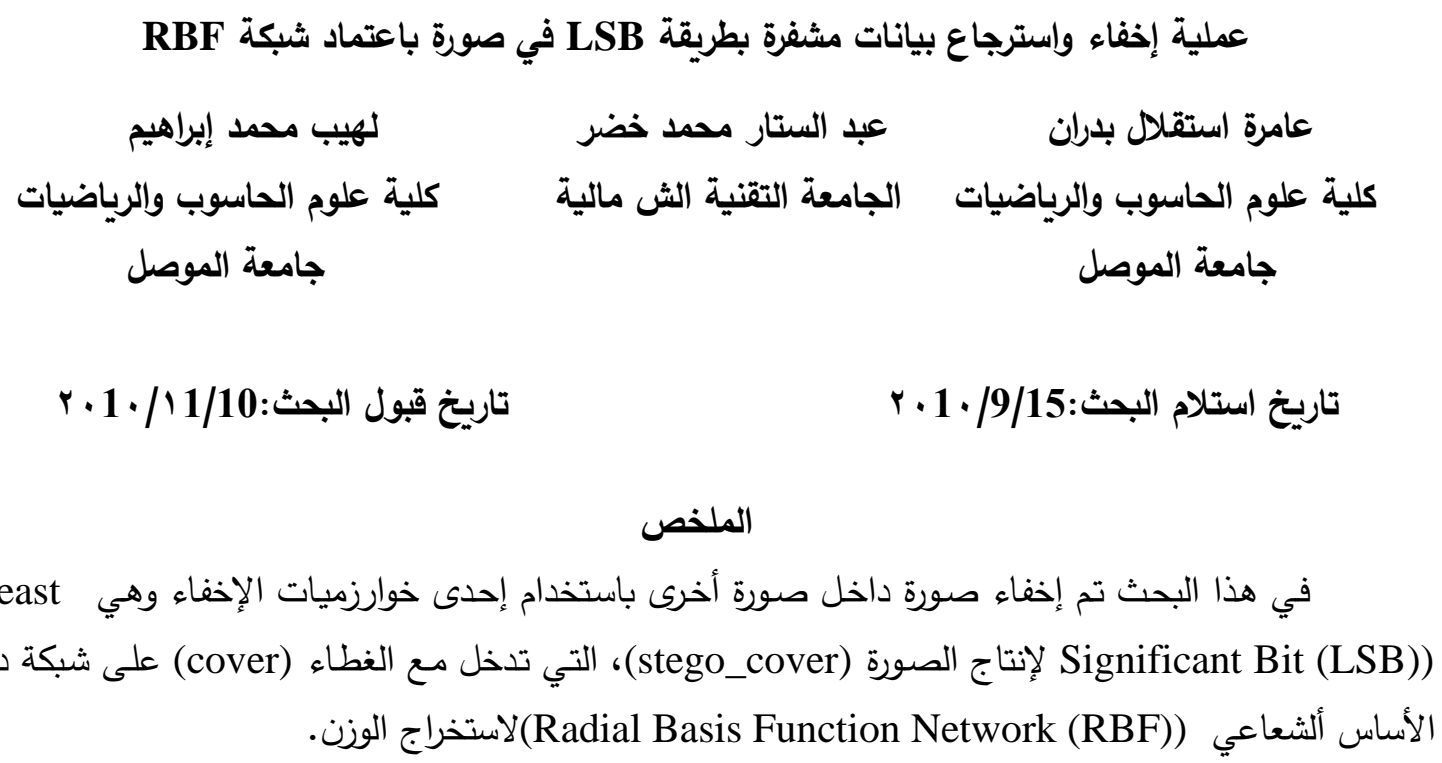


يـتم إرسـال الغطـاء لمـرة واحـدة إلـى المستلم ويمكن أن يحتفظ بـه لعـد غيـر محـدود مـن الرسـائل (messages). ولكل رسـالة يتم إخفاءها سوف يتم إرسـال الوزن (weight) فقط، والذي يرسل كمفتاح إلى المستلم, عندها يقوم المستلم باستخدام الغطاء مع الوزن الذي استلمه لفك الإخفاء، وبذلك فأن هذه الطريقة تتضمن مستويين من الحماية، المستوى الأول يمثل إخفاء الرسـالة في الغطاء لتكوين صورة مضمنه (stego-cover), والمستوى الثاني يمثل تثفير الصورة المضمنة باستخدام الثبكة العصبية (RBF) باعتبارها هي الهدف (target) والصورة الغطاء هي الإدخال إلى الثبكة، عندها يتم تكوين أوزان والتي تمثل البيانات المشفرة. وبعد ذلك بإمكان المستلم عن طريق شبكة (RBF) من فك الإخفاء والحصول على الصورة المضمنة (stego-cover) ومن ثم الحصول على الرسالة. ولقد تم استخدام لغة (Matlab R2008a) لاتجاز هذا البحث. الكلمات المفتاحية: تثفير ، خوارزمية LSB، شبكة RBF.

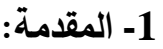

يهدف هذا البحث إلى المحافظة على سرية البيانات وعدم اطلاع المتطفل عليها وسهولة وصول المعلومة

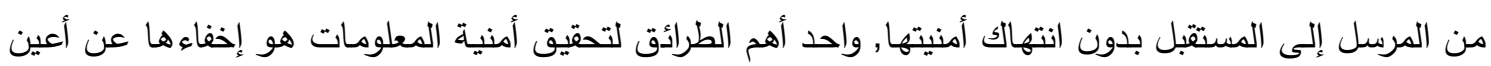

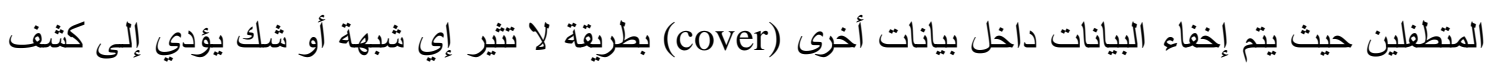

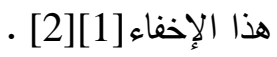

تم استخدام الثبكات العصبية الاصطناعية للمساعدة في إتمام عملية الإخفاء وفك الإخفاء ولإضافة درجة اكبر من الصـوبة لفك الإخفاء من قبل المتطفلين، وقد تم استخدام الشبكة العصبية (RBF) لاحتوائها على خاصية استرجاع البيانات بصورة صحيحة.

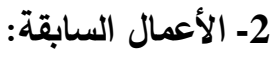

في البحث]3] تم أجراء عملية أخفاء للبيانات بدون تضمين البيانات وذلك عن طريق تدريب شبكة

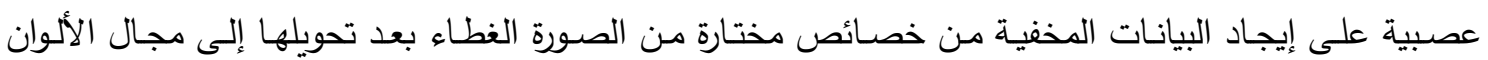

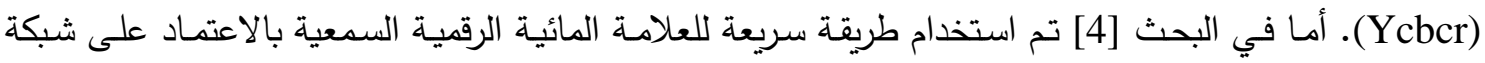

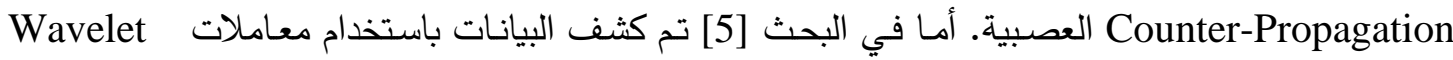
لصورة متضمنة للبيانات (stego-cover) وتدريب الثبكة العصبية عليها.

\section{3-1 شبكة دالة الأساس الشعاعي(Radial Basis Function Network)}

وتعد أكثر الثبكات استخداماً وهي أيضاً شبكة ذات تغذية أمامية لكن بطبقة مخفية واحدة. و) (RBF) هي

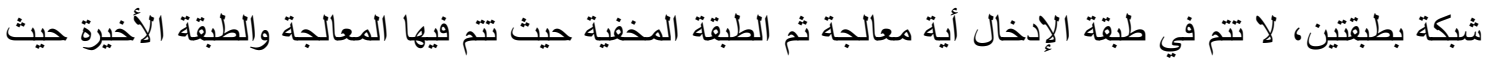

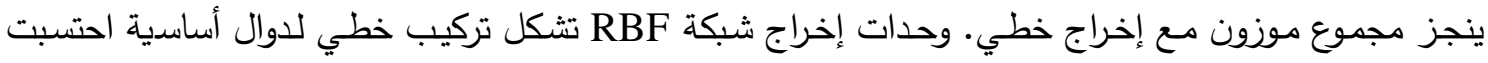


الدوال الأساسية في الطبقة المخفية تتتج استجابة محصورة في منطقة محددة للإدخال وهذه المنطقة لها مركز • وهذه الاستجابة تعد قيم إدخال خاصة ولها أعلى قيمة إخراج، وقيمة الإخراج هذه تصبح بمثابة قيم الإدخال

وتتطلق من هذه النقطة[7]. الدالة الأساسية نشير إليها كدالة تنشيط وهنا الدالة الأكثر شيوعاً هي دالة كاوس (Gaussian function) ، في

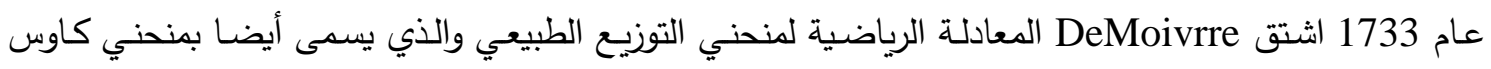
نسبة إلى (Gauss 1777-1855) الذي اشتق معادلته عند الخطأ في القياسات المتكررة] [8]. تأخذ الدالة شكل الجرس (bell shape) ، يعد التوزيع الطبيعي من التوزيعات المهمة والأكثر استخداماً

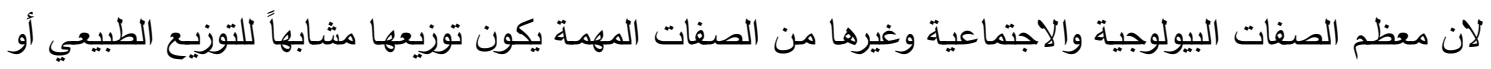

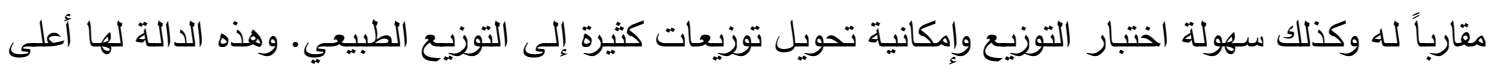
قيمة في نقطة المركز ثم تتناقص باتجاه الخارج[9][10]. دالة التنشيط لثبكة RBF هي (radial basis)، وكما موضح في المعادلة التالية[11]:

$$
\operatorname{radbas}(n)=e^{n^{2}}
$$

قيم الإخـراج للوحدة المخفيـة تقـع بين 0 و 1 ، الدخل الأقرب إلى مركز كـاوس هـي العقدة الأكبر استجابة(اكبر العقد استجابة) لان العقدة (العصب) تتتج إخراج مطابق للمدخلات بمسافة متساوية عن مركز كاوس وهذا ما يدعى بـ Radial basis [6][7]. والثكل رقم (1) يوضح فيه دالة التتشيط (11) (11).

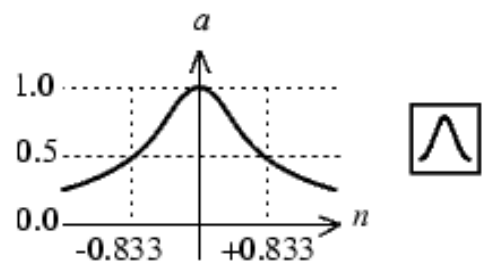

\section{الثكل(1): يوضح فيه دالة التنشيط}

شبكة RBF تثكل طبقة مخفية واحدة للدالة (Basis function) أو العصبونات. عند إدخال كل عصبون يتم حساب المسافة بين مركز العصبون وقيمة الإدخال ثم يتم تشكيل الإخراج للعصبون بتطبيق الدالة الأساسية (كاوس) لهذه المسافة. كذلك شبكات RBF يمكن أن تكون متعددة المخرجات. تحل شبكات RBF كثيراً من المشاكل وهي مفيدة للاستخدام في المسائل الآتية: أ- التقريب الدالي Function Approximation. ب- التصنيف Classification. ج- موديلات الأنظمة الديناميكية والسلاسل الزمنية[6]. 
تتألف شبكة RBF من بنية ذات تغذية أمامية مع طبقة إدخال وطبقة مخفية من وحدات شبكة RBF وطبقة إخراج مؤلفة من وحدات خطية. كما هو موضح في الثكل رقم(2).

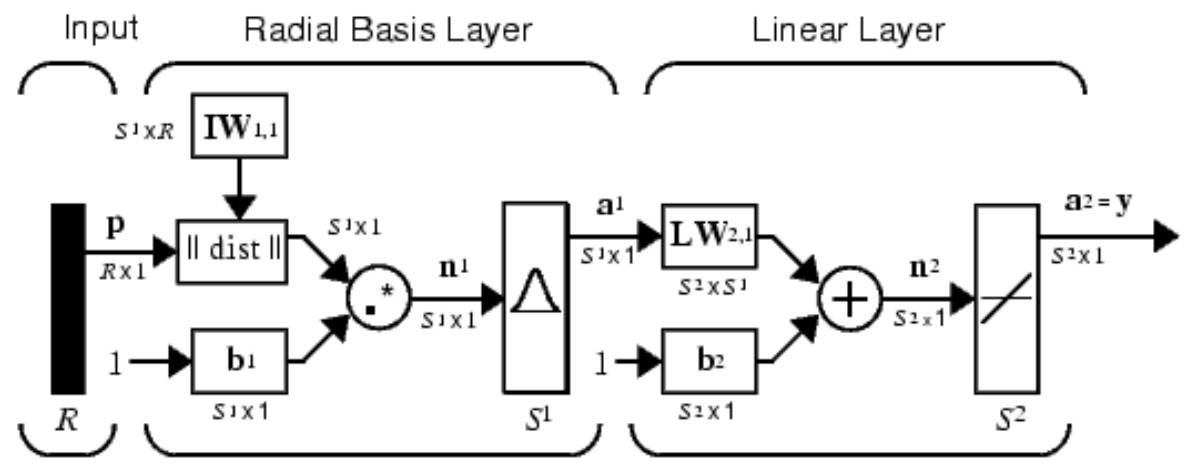

الثكل(2): يوضح فيه عمل شبكة RBF

$$
\begin{aligned}
& \text { Rد العناصر في عمود الإدخال. } \\
& \text { :عدد العصبونات في الطبقة الأولى.:S1 } \\
& \text { :عدد العصبونات في الطبقة الثانية. } \\
& \text { P } \\
& \text { IW : مصفوفة الوزن للطبقة المخفية. } \\
& \text { basis مصفوفة الـ b } \text { b }^{1}, b^{2} \\
& \text { : مصفوفة الوزن لطبقة الاخراج. LW" }
\end{aligned}
$$

تحول طبقة الإدخال متجه الإدخال إلى الوحدات المخفية التي تشكل استجابة محصورة لنمط الإدخال. تجززنا بمستويات التتشيط لوحدات الإخراج بإشارة الاقتراب لمتجه الإدخال إلى التصنيف. يمر التدريب بمرحلتين، حيث تستخدم تقنية العنقدة غير المُرشَدة (unsupervised clustering technique) للطبقة المخفية، بينما يطبق التدريب المُرشَد (supervised) على وحدات طبقة الإخراج[6]. العقد في الطبقة المخفية تنفذ بواسطة الدالة الأساسية التي تعدل على منطقة محصورة من حيز الإدخال. وذلك باعتماد المعادلة الآتية[11]:

$$
a l_{i}=\operatorname{radbas}\left(\left\|_{i} W_{1,1}-p\right\| b l_{i}\right)
$$

ومن ثم العقد في طبقة الإخراج تنفذ بواسطة الدالة الخطية التي تعمل على منطقة محصورة من حيز الإخراج. وذلك باعتماد المعادلة الآتية[11]:-

$$
a_{2}=\operatorname{purelin}\left(L W_{2,1} a_{1}+b_{2}\right)
$$

ثم يتم تحسين الثبكة بإضافة عقد انحياز (Bias node) إلى طبقة الإدخال والطبقة المخفية ويتم تغيير الوزن لهذه العقد كما هو الحال في بقية العقد المكونة للشبكة عدا قيمة الإدخال لعقدة الانحياز دائما تكون +6[1]. 


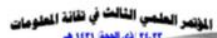

(2) 
في هذا النظام توجد لدينا صورتين الصورة الغطاء و message:-

4-الصورة الغطاء (cover):

في هذا البحث تم استخدام صورة ملونـة تتكون من ثلاث طبقات (R,G,B)، ممكن إن تكون الصورة

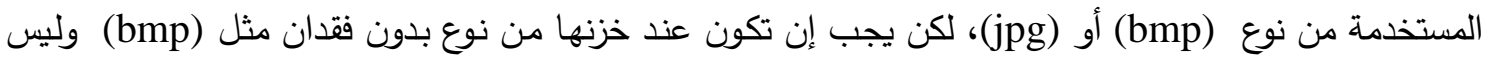

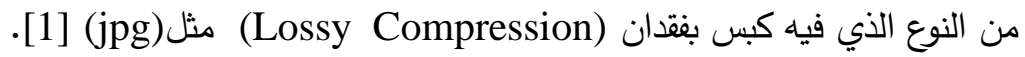

5- الصورة المراد إخفاءها (message):

الصورة المراد إخفاءها (message) يفضل إن تكون صورة ملونـة من نوع (bmp) ويفضل إن يكون

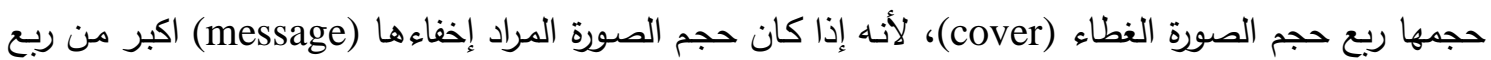
حجم (cover) فعندها سوف تكون نسبة التشوه اكبر ، لذلك سوف نختار الصورة (message) بحيث تكون ربع حجم الصورة (cover) ونقوم بتوزيع bits الصورة (message) داخل مصفوفة صفرية تكون بنفس إبعاد الصورة (cover)، وتكون عملية توزيع bits عند المرسل وقبل القيام بعملية الإخفاء ثم يعاد تجميع هذه bits عند المستلم بعد فك الإخفاء.

6 - 6 الطريقة المعتمدة عند المرسل :

هناك عدة خطوات يجب إتباعها عند المرسل، وكما هو موضح في الثكل (3).

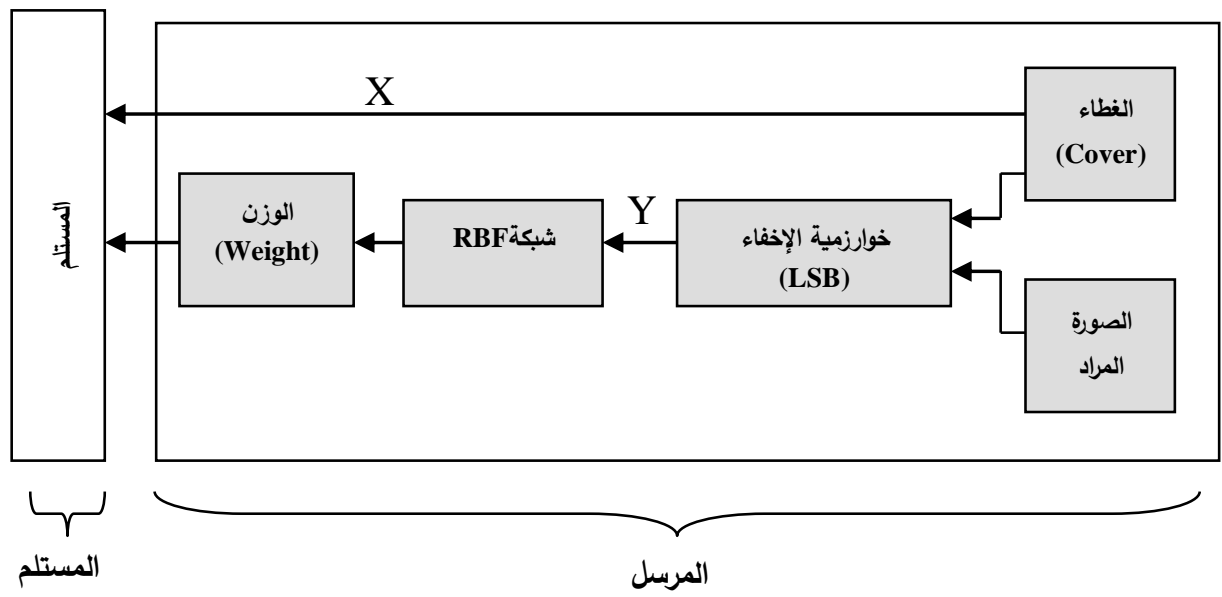

الثكل (3): مخطط خوارزمية الإخفاء

6-1 تهيئة الصورة عند المرسل:

يقوم النظـام بتكـوين مصـفوفة صـفرية إبعادهـا بـنفس إبعـاد الصـورة الغطــاء(cover) وتوزيـع (bits)

للصورة (message) على المصفوفة الصفرية، لجعل إبعاد الصورة (message) بنفس إبعاد الصورة (cover) وذلـك لان حجـم الصــورة (message) ربـع حجم الصـورة (cover)، إي إن كـل (2bits) مــن الصـورة يتم إخفاءها داخل (message) 
يتم توزيـع الصورة المـراد إخفاءهـا (message) على المصفوفة الصفرية، بحيث يتم توزيع bits كل (byte) من الصورة المراد إخفاءها (message) إلى (bytes (4 by ) من المصفوفة الصفرية، بحيث يحصل كل (byte)

بايت

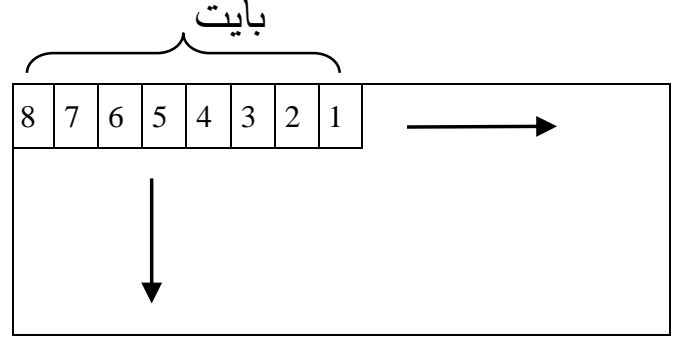

message الثكل(4-أ): الصورة

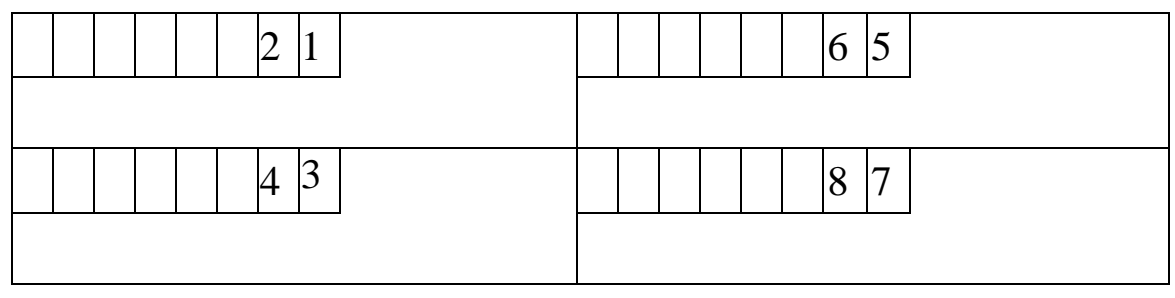

الثكل(4-ب): المصفوفة الصفرية

bits الشكل(4):عملية توزيع الـ

موضح في الثكل (4).

الخوارزميـة المستخدمة بعملية الإخفاء في هذا النظـام هي خوارزميـة البت الأقل أهمية, طريقـة عمل

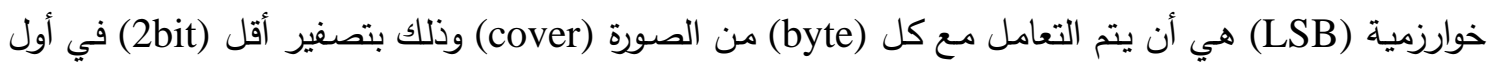
(byte)

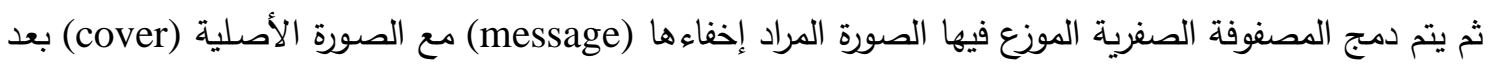
تصفير آخر (byte) (bits) من كلإنتاج (stego-cover). في هذا البحث تم استخدام خوارزمية (LSB) للإخفاء لبساطتها وسـهولة فهمها وتطبيقها، ويمكن استخدام أيـة خوارزمية أخرى للإخفاء بحسب ما يتوافق مع الغرض المستخدم له. -6-3- طريقة عمل شبكة RBF عند المرسل:

المدخل الأول لثبكة RBF هو الصـورة الغطاء (Cover) والذي يعتبر (X)، والمـذخل الثاني لشبكة

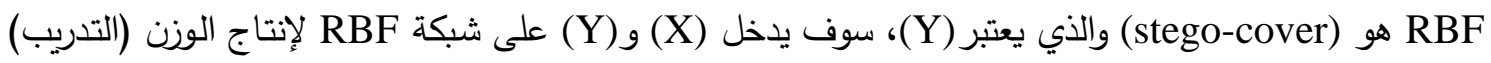
وذلك بالاعتماد على المعادلتين على التوالي (2) و(3)، ويتم إرسال الوزن و(cover) إلى الطرف الثاني (المستلم) لاستخدامه في استخراج الصورة (stego-cover). 


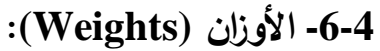

في هذه المرحلة الأوزان التي تم الحصول عليها لإرسالها إلى المستلم سوف تكون غير مفهومـة لأي مترصد، لذلك فهي تعتبر مرحلة مهمة من مراحل التشفير. 7- الطريقة المتبعة عند المستلم :

هناك عدة خطوات يجب إتباعها عند المستلم، وكما هو موضح في الثكل (5).

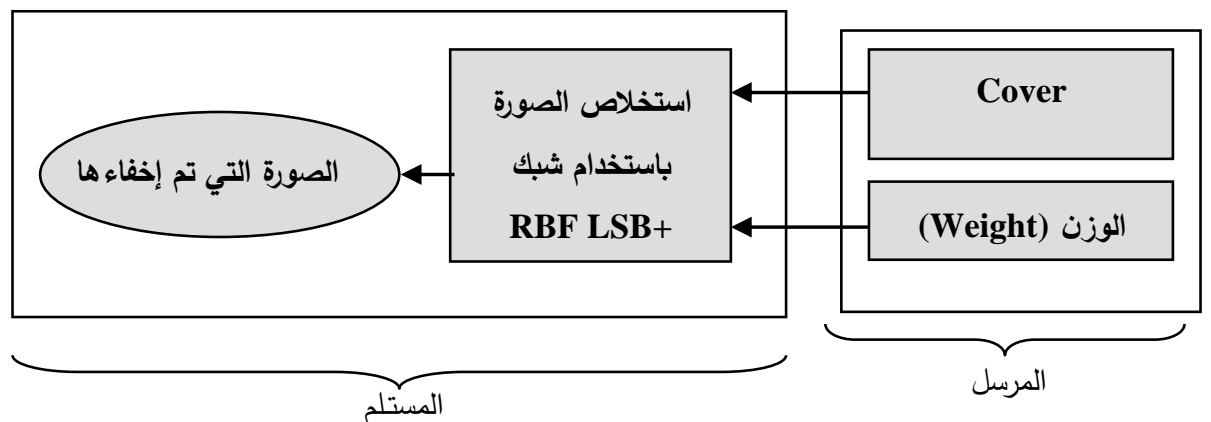

شكل(5): مخطط خوارزمية فك الإخفاء

RBF 7-1 طريقة عمل شبكة Rم المستلم:

بعد استلام الوزن (weight) والصسوة (cover) من المرسل، تقوم شبكة RBF باستخلاص الصسورة المخفية وذلك بالاعتماد على المعادلتين على التوالي (2)و(3)، وذلك لاستخراج الصورة (cover) من الثبكة.

7-2- عملية فك الإخفاء عند المستلم (التحليل):

عند إجراء عمليـة فك الإخفاء يتت طرح الصورة (stego-cover) من الصسورة (cover) لإنتاج الصـورة (message)، ثم يعاد تجميع التوزيع للصورة الناتجة من عملية الطرح.

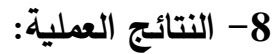

أ- الصـورة الغطـاء (cover) التي تـم تهيئتهـا لإخفـاء الصـورة (message) داخلهـا، كمـا هـو موضـح فـي

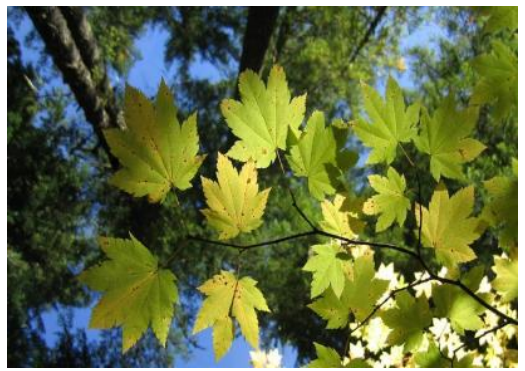

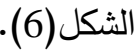

الثكل (6): الصورة الغطاء (cover) 
ب- الصـورة المراد إخفاءهـا (message)، التي تم تهيئتها لإخفائها داخل الصـورة الغطاء (cover)، كمـا هو موضح في الشكل(7).

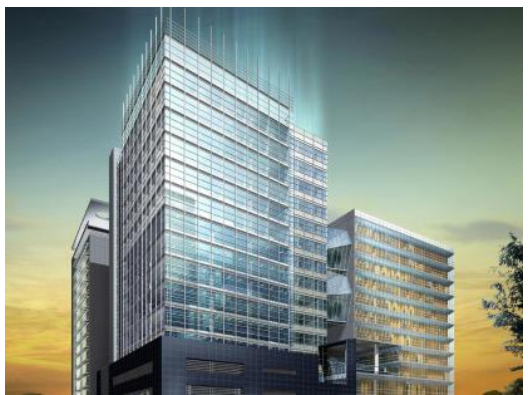

(الثكل (7): الصورة (message)

جـ.- بعـد إجـراء عمليـة الإخفـاء سـوف يـتم الحصـول علـى الصــورة الغطــاء (cover) وبـداخلها الصـورة (message) ) وتدعى (stego-cover) كما هو موضح في الثكل (8).

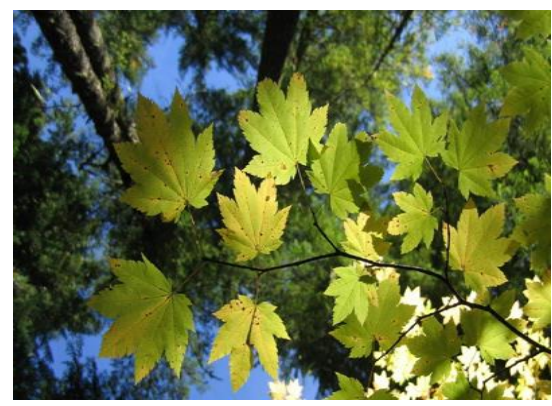

الثكل (8): نافذة الصورة (cover) مع الصورة (message) بعد عملية الإخفاء (stego-cover)

د- خزن الوزن (weight) الناتج من (stego-cover). هـ - بعدها يتم فك عملية الإخفاء, ليتم الحصول على الصورة (message) الأصلية فقط التي تم إخفاءها سابقاً داخل الصورة الغطاء (cover)، كما هو موضح في الثكل(9).

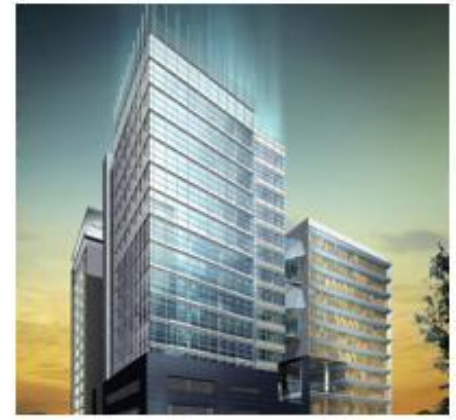

الثكل(9): الصورة (message) بعد عملية فالك الإخفاء 
يجب ملاحظـة ان المرسل سوف يرسل (Cover) إلى المستلم مـرة واحدة فقط وعندها سوف يحتفظ المستلم بال (Cover) لعدد غير محدود من الرسائل (messages). وكلما أراد المرسل إرسال (message) يرسل (med) ئرة فقط الوزن (Weight) عبر قنوات مغطاة بدون معرفة المنطفلين. وعندها سوف يقوم المستلم عن طريق (Cover) الثابت الذي سبق ان استلمه مع الوزن الذي سوف يتم الحصول عليه مؤخراً بفك الإخفاء والحصول على -stego) cover) ومـن ثم الحصـول على (message). ومـن الجدير بالذكر انسه يمكن ارسـال (Cover) بقنـاة عاديـة و) بقناة مغطاة إذا رغب المرسل بذلك.

ويجب ملاحظة انـه عدد الإدخالات بعدد اسطر (Cover)، وعدد الاخراجات بعدد اسطر -(stego)

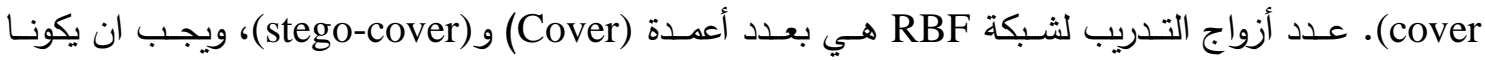
متساويين، الثكل رقم (10) يوضح فيه عملية التدريب لشبكة RBF.

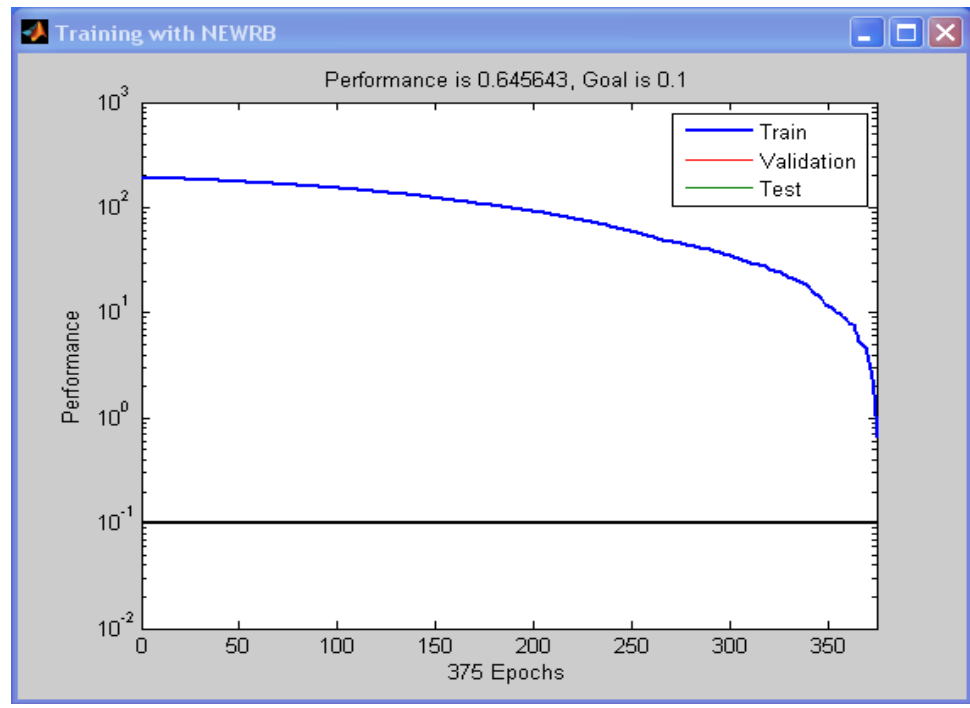

الشكل(10): يوضح عملية التدربب لشبكة RBF

فائدة استخدام (Stego-cover) قرببة من (Cover) بحيث لا يمكن اكتثاف ان فيها بيانات مخفية بالعين المجردة بحيث يصعب على المتطفل اكتثاف البيانات المخفية حتى في حالة حصوله على (Cover) والأوزان. بالإضافة إلى انه يفضل استخدام صورة كثيرة التفاصيل بالنسبة (Cover)، بحيث لا يكون فيها أي عمودين متثابهين.

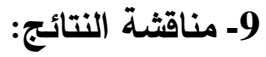
تم إيجاد نتائج (PSNR) لـ(50) مثال وتم حساب(Mean Square Error (MSE) وكانت النتيجة انه تم استرجاع جميع البيانات المشفرة بدون فقدان. هذه الطريقة تتضمن مستويين من الحماية، المستوى الأول يمثل إخفاء الرسالة في الغطاء لتكوين صورة مضـنه (stego-cover), والمستوى الثاني يمثل تثنفير الصـورة المضــنة باستخدام الثـبكة العصبية (RBF) باعتبارها هي الهدف (target) والصسورة الغطاء هي الإدخال إلى الثبكة, عندها يتم تكوين أوزان والتي تمثل البيانات المشفرة، التي تستخدم فيما بعد عند المستلم مع الغطاء لإيجاد البيانات الأصلية. 


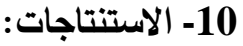

$$
\begin{aligned}
& \text { أ- يمكن إخفاء حجم كبير من البيانات دون التأثير على ألوان الغطاء. } \\
& \text { ب- عند استرجاع الرسالة (message) تسترجع بدون فقدان. } \\
& \text { ج- استخدام الشبكات العصبية أعطت أمنية عالية في الإخفاء . } \\
& \text { المصادر } \\
& \text { برزنجي, فوزي, (2008)، "إخفاء البيانات داخل الصورة"، جامعة السليمانية، العراق. }
\end{aligned}
$$

[2]. A., Muhalim M., (2003), "Information Hiding Using Steganography", University Technology Malaysia.

[3]. K. Naoe, Takefuji, (2008), "Damage less Information Hiding using Neural network on Ycbcr Domain", IJCSNS, Vol 8 No. 9, September.

[4]. W. Guohua, (2008), "A Fast Audio Digital Watermark Method Based on Counter-Propagation Neural Net Works", Hangzhou Dianzi University Institute of Graphics and Image Hangzhou, Chin 9.

[5]. D. Jennifer, B. Clifford, (2005), "An Artificial Neural Network for Wavelet Steganalysis", Lowa State University, Ames Iowa, 50011.

[6] حسن، سوزان، (2007)، "استخدام الثبكتين العصبيتين الاصطناعيتين شبيهة نيوتن ودالة الأساس

$$
\text { ألثعاعي في تشخيص مرض خلع الورك الولادي"، جامعة تكريت. }
$$

[7]. Patterson D. (1996), "Artificial Networks", Singapore, Prentice Hall.

$$
\text { الراوي، د.خاشع محمود (1984)، "المدخل إلى الإحصاء"، مطابع جامعة الموصل. }
$$

[9]. Huang J. Shimizu A. (2002), "Robust Face Detection Using A Modified Radial Basis Function Network", IEICE Trans. ,Inf. \& Syst. Vol E85-D,No 10.

[10]. Pao, Y.H., (1998), "Adaptive Pattern Recognition And Neural Networks", Wesley Publishing Company, Inc. New York.

[11]. Howared D., Mark B., (2008), "Neural Network toolbox user's", The mathworks. 